\section{Hier steht eine Anzeige.}

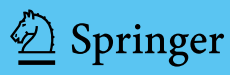

\title{
Zuweisungen in ein epilepsiechirurgisches Programm erfolgen zu zögerlich
}

\begin{abstract}
Trotz eindeutiger Indikationskriterien ist es klinische Realität, dass ein epilepsiechirurgisches Monitoring häufig erst nach jahrzehntelanger Erkrankung erfolgt. Zwei aktuelle Studien gehen den Ursachen dafür auf den Grund.
\end{abstract}

$D^{i}$ ie Empfehlungen der AAN sind kurz und klar [1]: Nach Versagen des zweiten Medikamentes, einzeln oder in Kombination, ist eine epilepsiechirurgische Evaluation durchzuführen, unabhängig von der aktuellen Anfallsfrequenz oder Dauer der Erkrankung. Trotz dieser eindeutigen Indikationskriterien ist es klinische Realität, dass ein epilepsiechirurgisches Monitoring häufig erst nach jahrzehntelanger Erkrankung erfolgt. Zwei aktuelle Studien suchen nach den Ursachen für die zögerliche Zuweisungspraxis.

In einer kanadischen Studie wurden 327 Neurologen mittels Fragebogen zu Wissen und Einstellung zu Epilepsiechirurgie befragt [2]. Die Ergebnisse verwiesen nach Meinung der Autoren auf deutliche Wissenslücken zu den Prinzipien epilepsiechirurgischer Abklärung und Behandlung: Beispielsweise forderten über die Hälfte der befragten Neurologen eine minimale Anfallsfrequenz von mindestens einem Anfall pro Jahr, knapp die Hälfte der Teilnehmer konnten die Kriterien der Pharmakoresistenz nicht korrekt benennen und nur zirka $54 \%$ gaben an, dass bei nachgewiesener Pharmakoresistenz eine prächirurgische Evaluation schnellst möglich erfolgen sollte. Neben der häufig fehlenden Kenntnis der Empfehlungen in Bezug auf Epilepsiechirurgie waren wahrgenommene Engpässe im Versorgungssystem ein weiterer Grund für zu zögerliche Zuweisungen der Patienten in ein epilepsiechirurgisches Programm.

Die Übersichtsarbeit von Dewar et al. [3] beleuchtet die Einstellungen von $\mathrm{Pa}$ tienten und Ärzten zur Epilepsiechirurgie. Einzelstudien werden kurz vorgestellt und deren Ergebnisse in ein theoretisches Modell der Entscheidungsfindung integriert. Während medikamentöse Entscheidungen häufig komplett den Ärzten überlassen werden, erfolgt die Entscheidung für einen epilepsiechirurgischen Eingriff unter enger Abspra- che mit dem Patienten. Dabei besteht angesichts einer Behandlungsoption mit unsicherem aber irreversiblem Outcome ein schwer auflösbarer Konflikt - sowohl auf Seiten der Patienten wie auch der Behandler. Dahinter stehen Furcht vor einer Operation am Gehirn, Unwissen hinsichtlich nichtmedikamentöser Behandlungsoptionen und eine parallel zur Erkrankungsdauer zunehmende Toleranz der Symptomatik. Anhand des Modells lassen sich verschiedene Maßnahmen ableiten, die eine Entscheidungsfindung zugunsten einer Operation erleichtern könnten - Evidenzen für deren Wirksamkeit gibt es jedoch noch nicht.

\section{Kommentar}

Zur Ursache für die bisher allzu zögerliche Zuweisungsrate zu epilepsiechirurgischen Interventionen verweist die Studie von Roberts auf unzureichende Kenntnisse auf Seiten der behandelnden Ärzte, die Übersichtsarbeit von Dewar ergänzt dies mit einem eher psychologischen Blick auf den gemeinsamen Entscheidungskonflikt in Anbetracht vielfältiger Unsicherheiten und Befürchtungen. Inwieweit die Ergebnisse der Studien international übertragbar sind, ist unklar. Lange Zeitintervalle zwischen Erkrankungsbeginn und Operation finden sich aber auch in epilepsiechirurgischen Studien deutscher Zentren. Zur Verbesserung dieser Situation kommt insbesondere den Leitlinien der Fachgesellschaft eine zentrale Rolle zu. Diese sollten klar formuliert sein und möglichst nicht in Widerspruch zu internationalen Empfehlungen stehen.

Dr. Martin Lutz

1. Engel J Jr et al. Practice parameter: temporal lobe and localized neocortical resections for epilepsy. Epilepsia 2003; 44 (6): 741-51

2. Roberts $\mathrm{Jl}$ et al. Neurologists' knowledge of and attitudes toward epilepsy surgery: a national survey. Neurology 2015; 84 (2): 159-66

3. Dewar SR et al. Perceptions of epilepsy surgery: a systematic review and an explanatory model of decision-making. Epilepsy Behav 2015; 44: 171-8 